\title{
A PRIORI ESTIMATES AND EXISTENCE FOR A CLASS OF FULLY NONLINEAR ELLIPTIC EQUATIONS IN CONFORMAL GEOMETRY
}

\author{
XU-JiA WANG \\ The Australian National University
}

\begin{abstract}
In this paper we prove the interior gradient and second derivative estimates for a class of fully nonlinear elliptic equations determined by symmetric functions of eigenvalues of the Ricci or Schouten tensors. As an application we prove the existence of solutions to the equations when the manifold is locally conformally flat or the Ricci curvature is positive.
\end{abstract}

Dedicated to the memory of Professor S.S. Chern

\section{Introduction}

Let $\left(\mathcal{M}, g_{0}\right)$ be a compact Riemannian manifold of dimension $n \geq 3$. Denote by $\left[g_{0}\right]$ the set of metrics conformal to $g_{0}$. For $g \in\left[g_{0}\right]$ we denote by $R, R i c$, and $A_{g}=\frac{1}{n-2}\left(\right.$ Ric $_{g}-$ $\left.\frac{R_{g}}{2(n-1)} g\right)$ the scalar curvature, the Ricci tensor, the Schouten tensor, respectively. Let $\lambda\left(A_{g}\right)=\left(\lambda_{1}, \cdots, \lambda_{n}\right)$ denote the eigenvalues of $A_{g}$ with respect to $g$. In this paper we study the regularity and existence of solutions to the equation

$$
f(\lambda)=\varphi,
$$

where $\varphi$ is a positive, smooth function.

When $f=\sum \lambda_{i}$, equation (1.1) is the prescribing scalar curvature equation. When $\varphi \equiv 1$, it is the Yamabe problem. In this paper we assume that $f$ is a nonlinear function defined on an open convex cone $\Gamma \subset \mathbf{R}^{n}$ and satisfying the following conditions:

(f1) $f>0$ in $\Gamma$ and $f=0$ on $\partial \Gamma$;

(f2) $f_{i}>0$ in $\Gamma$, where $f_{i}$ denotes derivative in the $i$ th variables;

(f3) $f$ is concave;

(f4) $f$ is invariant under exchange of variables;

(f5) $f$ is homogeneous of degree $\alpha$ for some $\alpha>0$, namely $f(t \lambda)=t^{\alpha} f(\lambda) \forall t>0$.

This work was supported by the Australian Research Council.

Published in Chinese Annals of Mathematics, 27(B) (2006), 169-178. 
By the concavity, we have $\alpha \in(0,1]$. Note that (f1) and (f5) also implies (f2). If $\varphi \equiv 1$, then (f5) (with $\alpha=1$ ) also follows from (f1)-(f4), as one can define a new function $\tilde{f}$ such that $\left\{\lambda \in \mathbf{R}^{n} \mid \tilde{f}(\lambda)=1\right\}=\left\{\lambda \in \mathbf{R}^{n} \mid f(\lambda)=1\right\}$ [LL2]. We assume the cone $\Gamma$ satisfies

(g1) $\Gamma_{n} \subset \Gamma \subset \Gamma_{1}$, where $\Gamma_{1}$ and $\Gamma_{n}$ are given in (1.3) below;

(g2) if $\lambda \in \Gamma$, then any permutation of $\lambda$ also lies in $\Gamma$.

Equation (1.1) is referred to as conformal Hessian equation. A related equation is the Hessian equation, that is when $\lambda$ are eigenvalues of the Hessian matrix $\nabla^{2} u$. The Hessian equation was first studied in [CNS, I]; see also [CW, TW1] for further results. There are many functions satisfy the above conditions, for example,

(i) The conformal $k$-Hessian equation. Let

$$
\sigma_{k}(\lambda)=\sum_{i_{1}<\cdots<i_{k}} \lambda_{i_{1}} \cdots \lambda_{i_{k}}
$$

be the $k$-th elementary symmetric polynomial and let $f(\lambda)=\sigma_{k}^{1 / k}(\lambda)$. The corresponding cone is given by

$$
\Gamma_{k}=\left\{\lambda \in \mathbf{R}^{n} \mid \sigma_{j}(\lambda)>0 \text { for } j=1, \cdots, k\right\} .
$$

Note that $\Gamma_{1}$ is the half-space $\left\{\lambda \in \mathbf{R}^{n} \mid \sum \lambda_{i}>0\right\}$ and $\Gamma_{n}$ is the positive cone $\{\lambda \in$ $\left.\mathbf{R}^{n} \mid \lambda_{i}>0 \quad \forall 1 \leq i \leq n\right\}$.

The quotient equation

$$
f(\lambda)=\left(\frac{\sigma_{k}}{\sigma_{l}}\right)^{1 /(k-l)}(\lambda)
$$

also satisfies (f1)-(f5), where $0 \leq l<k \leq n$ and $\Gamma=\Gamma_{k}$.

(ii) For any integer $1 \leq k \leq n$ and $\delta \geq 0$,

$$
f(\lambda)=\delta \sum_{i=1}^{n} \lambda_{i}+\min \left\{\lambda_{i_{1}}+\cdots+\lambda_{i_{k}} \mid 1 \leq i_{1}<\cdots<i_{k} \leq n\right\}
$$

and $\Gamma=\left\{\lambda \in \mathbf{R}^{n} \mid f(\lambda)>0\right\}$. When $\delta>0$, this is indeed the Pucci operator, and is uniformly elliptic $[\mathrm{GT}]$.

(iii) More functions satisfy the above conditions, such as

$$
f(\lambda)=\left(\sum_{i=1}^{n} \lambda_{i}^{-2}\right)^{-1 / 2}
$$

and

$$
f(\lambda)=\left[\sum \lambda_{1}^{-\alpha_{1}} \cdots \lambda_{n}^{-\alpha_{n}}\right]^{-1 / k},
$$

where the sum is taken over all nonnegative integers $\alpha_{1}, \cdots, \alpha_{n}$ with $\sum \alpha_{i}=k$, and $\Gamma=\Gamma_{n}$, see [G]. One can find more functions satisfying the above conditions [Kr].

(iv) Let $f_{1}, f_{2}$ satisfy (f1)-(f5) and $f_{2}$ is homogeneous of degree 1 . Let

$$
f(\lambda)=f_{1}\left(\lambda+\delta f_{2}(\lambda) e\right),
$$


where $\delta>0$ is a constant, $e=(1, \cdots, 1)$.

From the definition of Schouten tensor, the Ricci curvature $\mu=\left(\mu_{1}, \cdots, \mu_{n}\right)$ is given by

$$
\mu_{i}=\lambda_{i}+\frac{1}{n-2} \sum \lambda_{i}
$$

Hence if we choose $\delta=\frac{1}{n-2}$ and $f_{2}(\lambda)=\sum \lambda_{i}$ in (1.8), then we get an equation for the Ricci curvature $\mu=\left(\mu_{1}, \cdots, \mu_{n}\right)$,

$$
f_{1}(\mu)=1
$$

To write (1.1) as a partial differential equation, we use the conformal transformation

$$
g=v^{\frac{4}{n-2}} g_{0}
$$

Then (1.1) becomes

$$
f\left(\lambda\left(A^{v}\right)\right)=\varphi
$$

where

$$
A^{v}=\frac{2}{n-2} v^{-\frac{n+2}{n-2}}\left(\nabla_{c o n f}^{2} v+\frac{n-2}{2} v A_{g_{0}}\right) .
$$

and

$$
\nabla_{c o n f}^{2} v=-\nabla^{2} v+\frac{n}{n-2} \frac{\nabla v \otimes \nabla v}{v}-\frac{1}{n-2} \frac{|\nabla v|^{2}}{v} I
$$

In this paper we call $\nabla_{\text {conf }}^{2} v$ the conformal Hessian matrix.

Equation (1.11) is a fully nonlinear partial differential equation, in order that it is elliptic, we assume that the eigenvalues $\lambda \in \Gamma$. Accordingly we say a metric $g \Gamma$-admissible, or simply admissible, if $\lambda \in \Gamma$. Denote by $\left[g_{0}\right]_{\Gamma}$ the set of all conformal admissible metrics,

$$
\left[g_{0}\right]_{\Gamma}=\left\{g \in\left[g_{0}\right] \mid \lambda\left(A_{g}\right) \in \Gamma\right\}
$$

When $g$ is an admissible solution to (1.1), the function $\varphi$ must be positive. In the following we will consider solutions with $\lambda\left(A_{g}\right) \in \Gamma$ only.

The existence of admissible solutions to (1.1) has been studied by many authors and most papers are concentrated on the conformal $k$-Hessian equation. Just to mention a few, the first existence result is by [CGY], where the existence of solutions was proved for the case $k=2, n=4$. Subsequently [LL1], [GW2] proved the existence of solutions for general $k$ and $n$, but on locally conformally flat manifolds. In [GV1] the authors proved the existence for $k>\frac{n}{2}$, provided $\mathcal{M}$ is not conformal to the unit sphere.

Recently with Sheng and Trudinger [STW], we proved the existence of solutions to the conformal $k$-Hessian equation for $k \leq \frac{n}{2}$, provided equation (1.1) is variational. In [TW2] we proved the compactness of the set $\left[g_{0}\right]_{\Gamma_{k}}$ for $k>\frac{n}{2}$, for any compact manifold not conformal to $S^{n}$, which also yields the existence of solutions to the conformal $k$-Hessian equation for $k>\frac{n}{2}$. Note that in [CGY, LL1, GW2, STW] the existence was proved for $\varphi \equiv 1$. 
In this paper we establish the interior a priori estimates for solutions to (1.1) under the mild conditions (f1)-(f5). The interior a priori estimates were proved for the conformal $k$-Hessian equation by P. Guan and G. Wang [GW1]. They also proved the interior estimates for the quotient equation (1.4). Our proof here uses a blow-up argument and is based on the Liouville theorem of Y.Y. Li [L] and applies to the general equation (1.1) with $f$ satisfying (f1)-(f5). From the a priori estimates, we prove the existence of solutions of (1.1) when $\mathcal{M}$ is locally conformally flat and $\varphi \equiv 1$, or when the cone $\Gamma$ is smaller than $\Gamma_{n / 2}$. In the latter case, the Ricci curvature must be positive.

This paper is arranged as follows. In Section 2 we state our main results. In Section 3 we prove the interior second derivative estimate. In Section 4 we prove the interior gradient estimate. The final section 5 discusses that existence of solutions to (1.1).

In a workshop at Berkeley in November 2005, the author learnt that Sophie Chen has independently proved the interior a priori estimates [Ch], see Remark 2.1 for more details. The author would also like to thank Viaclovsky for discussions on Theorems 2.3 and 2.4 at the workshop.

\section{Main results}

For the a priori estimates in Theorems 2.1 and 2.2 below, we assume that $\varphi$ is a nonnegative function satisfying $\nabla^{2} \varphi \geq-C$ for some constant $C>0$.

Theorem 2.1. Let $v \in C^{3}$ be an admissible positive solution of (1.11) in a geodesic ball $B_{r}(0) \subset \mathcal{M}$. Then

$$
\frac{|\nabla v|}{v}(0) \leq C
$$

where $C$ depends only on $n, r, \varphi, f, \sup v$, and $g_{0}$, and $\nabla$ denotes the covariant derivative with respect to $g_{0}$.

Theorem 2.2. Let $v \in C^{4}$ be an admissible positive solution of (1.11) in $B_{r}(0)$. Then

$$
\left|\nabla^{2} v\right|(0) \leq C
$$

where $C$ depends only on $n, r, \varphi, f, \inf v, \sup (v+|\nabla v|)$.

Remark 2.1. As indicated in the introduction, the author learnt in a workshop at Berkeley in early November 2005 (31/10-4/11, 2005) that Sophie Chen [Ch] has independently proved Theorems 2.1 and 2.2. Her preprint was available in September 2005, while the first version of the paper was finished in October before the workshop. The proofs in [Ch] and in this paper are completely different. The proof in this paper uses a blow-up argument and the Liouville theorem [L], while the estimates in [Ch] are derived directly from an auxiliary function and so are more favourable. 
Once the second derivatives are bounded, the equation becomes uniformly elliptic if $\varphi$ is positive, and further regularity follows from the Evans-Krylov regularity theory.

The proof of the second derivative estimate is similar to that in [GW1,GW3,STW]. For the interior gradient estimate, we use a different approach. An important property of the conformal Hessian equation is its invariance under Kelvin transformation (when the equation is defined in $\mathbf{R}^{n}$ ). Hence by the moving plane method, Y. Li [L] proved the Liouville theorem for the equation

$$
F\left(\nabla_{\text {conf }}^{2} v\right)=0 \text { in } \mathbf{R}^{n}
$$

That is an entire positive solution of (2.3) must be a constant. Using a blow-up argument, we prove the gradient estimate by the Liouville theorem and the interior second derivative estimate.

As an application, we prove the existence of solutions to equation (1.1) when $\mathcal{M}$ is locally conformal flat or the cone $\Gamma$ is smaller that $\Gamma_{n / 2}$.

Theorem 2.3. Let $\left(\mathcal{M}^{n}, g_{0}\right)$ be a compact, locally conformally flat manifold. Suppose $\varphi \equiv 1$. Then there exists a solution to problem (1.1).

Remark 2.2. The existence of solutions to problem (1.1), for general $f$ and $\varphi \equiv 1$, was also proved in [LL2] (see Theorem 1.1 in [LL2]).

We also remark that if $\varphi$ is not a constant, there are obstructions to the existence of solutions in general.

In our next existence result, we assume the cone $\Gamma$ is relative small, so that any admissible metric has positive Ricci curvature. For this purpose we introduce a cone $\Sigma_{\delta}$ as in [GV2], that is

$$
\Sigma_{\delta}=\left\{\lambda \in \mathbf{R}^{n} \mid \min \lambda_{i}+\delta \sum \lambda_{i}>0\right\}
$$

where $\delta \geq 0$. From (1.9),

$$
\operatorname{Ric}_{g} \geq 0 \text { if and only if } \lambda\left(A_{g}\right) \in \Sigma_{\frac{1}{n-2}},
$$

and Ric $_{g}>0$ if and only if $\lambda\left(A_{g}\right) \in \Sigma_{\delta}$ with $\delta<\frac{1}{n-2}$, except when $g$ is locally a flat metric. Taking $l=1$ in the proof of Lemma 4.2 in [TW1], we also have

$$
\Gamma_{k} \subset \Sigma_{\frac{n-k}{n(k-1)}} .
$$

In particular we have Ric $_{g} \geq 0$ if $\lambda\left(A_{g}\right) \in \Gamma_{n / 2}$ and Ric $_{g}>0$ if $\lambda\left(A_{g}\right) \in \Gamma_{k}$ for $k>\frac{n}{2}$.

Theorem 2.4. Let $\left(\mathcal{M}^{n}, g_{0}\right)$ be a compact manifold not conformally equivalent to the unit sphere. Suppose $\varphi$ is a positive smooth function and the cone $\Gamma \subset \Sigma_{\delta}$ for some $0 \leq \delta<\frac{1}{n-2}$. Then there exists a solution to problem (1.1).

Remark 2.3. Theorem 2.4 was first proved by Gursky and Viaclovsky [GV1] for the conformal $k$-Hessian equation for $k>\frac{n}{2}$, see also [TW2] for a different proof. By the 
very recent a priori estimates in [Ch], Gursky and Viaclovsky extended their existence result to general curvature function $f$ as in Theorem 2.4 [GV1].

Remark 2.4. In Theorems 2.3 and 2.4, we will not only prove the existence of solutions but also the compactness of the set of solutions if $\left(\mathcal{M}, g_{0}\right)$ is not conformally equivalent to the unit sphere. In particular under the assumptions in Theorem 2.4, the set of all admissible metrics (subject to the volume constraint $\mathrm{Vol}_{\mathcal{M}}=1$ ) is compact.

\section{Second derivative estimate}

Denote

$$
F\left(A^{v}\right)=f\left(\lambda\left(A^{v}\right)\right),
$$

where $A^{v}$ is the matrix given in (1.12). Regard $F$ as a function of $n \times n$ real symmetric matrices, $F=F\left(a_{i j}\right)$. Then from [CNS], we know that $F$ is elliptic by (f2), concave by (f3), and symmetric under orthogonal transformations by (f4). That is

$$
\left\{F^{i j}\right\}:=\left\{\frac{\partial F}{\partial a_{i j}}\right\}>0
$$

for any matrix $A=\left(a_{i j}\right)$ with eigenvalues in $\Gamma$,

$$
F^{i j, s t}:=\frac{\partial^{2} F}{\partial a_{i j} \partial a_{s t}} b_{i j} b_{s t} \leq 0
$$

for any matrix $B=\left(b_{i j}\right)$, and

$$
F\left(O A O^{\prime}\right)=F(A)
$$

for any orthogonal matrix $O$.

For the a priori estimates for the second derivatives, it is convenient to use the conformal transformation $g=u^{-2} g_{0}$. Then

$$
A_{g}=\frac{u_{i j}}{u}-\frac{|\nabla u|^{2}}{2 u^{2}} g_{0}+A_{g_{0}}
$$

and equation (1.1) becomes

$$
F(U)=\varphi u^{-k \alpha}
$$

where

$$
U=\left\{u_{i j}-\frac{|\nabla u|^{2}}{2 u} g_{0}+u A_{g_{0}}\right\}
$$

Let $u \in C^{3.1}$ be an admissible positive solution of $(3.5)$ in a geodesic ball $B_{r}(0) \subset \mathcal{M}$. We want to prove

$$
\left|\nabla^{2} u\right|(0) \leq C
$$


where $C$ depends only on $n, r, \sup \left(u+u^{-1}\right), \sup |\nabla u|$, and $g_{0}$.

Let

$$
z=\rho^{2} u_{\xi \xi}
$$

where $\xi$ is any unit tangential vector, $u_{\xi \xi}=u_{i j} \xi_{i} \xi_{j}, \rho(x)=\left(1-\frac{|x|^{2}}{r^{2}}\right)^{+}$is a cut-off function, $|x|$ denotes geodesic distance from 0 . Assume that $\sup z$ is attained at $x_{0}$ and in direction $e=(1,0, \cdots, 0)$. In an orthonormal frame at $x_{0}$, we may assume by a rotation of axes that $\tilde{u}_{i j}:=u_{i j}+u a_{i j}$ is diagonal. Then at $x_{0}, F^{i j}$ is diagonal and

$$
\begin{aligned}
& 0=(\log z)_{i}=\frac{2 \rho_{i}}{\rho}+\frac{\tilde{u}_{11, i}}{\tilde{u}_{11}}, \\
& 0 \geq(\log z)_{i i}=\left(\frac{2 \rho_{i i}}{\rho}-\frac{6 \rho_{i}^{2}}{\rho^{2}}\right)+\frac{\tilde{u}_{11, i i}}{\tilde{u}_{11}} .
\end{aligned}
$$

Next, differentiating equation (3.5) twice, we obtain

$$
F^{i j} U_{i j, k k}=-\frac{\partial^{2} \mu\left(\sigma_{k}(\lambda(U))\right)}{\partial U_{i j} \partial U_{r s}} U_{i j, k} U_{r s, k}+\nabla_{k}^{2}\left(\varphi u^{-k \alpha}\right) \geq \nabla_{k}^{2}\left(\varphi u^{-k \alpha}\right),
$$

where $U_{i j, k}=\nabla_{k} U_{i j}$. Assume that

$$
\frac{|\nabla u|}{u} \leq C
$$

Then by (3.7) and the Ricci identities,

$$
U_{i j, 11}=u_{i j 11}-\frac{u_{k 1}^{2}}{u} \delta_{i j}+O\left(\frac{1+u_{11}}{\rho}\right)=u_{11 i j}-\frac{u_{k 1}^{2}}{u} \delta_{i j}+O\left(\frac{1+u_{11}}{\rho}\right) .
$$

Hence we obtain

$$
\begin{aligned}
0 & \geq \sum_{i} F^{i i}(\log z)_{i i} \geq-\frac{C}{\rho^{2}} \mathcal{F}+F^{i i} \frac{\tilde{u}_{11, i i}}{\tilde{u}_{11}} \\
& \geq-\frac{C}{\rho^{2}} \mathcal{F}+\frac{u_{11}^{2}}{u \tilde{u}_{11}} \mathcal{F}+\frac{1}{\tilde{u}_{11}} \nabla_{1}^{2}\left(\varphi u^{-k \alpha}\right),
\end{aligned}
$$

where $\mathcal{F}=\sum F^{i i}$.

Note that for any constant $a \geq 0$, there exists a constant $C_{a}>0$ such that $\mathcal{F}(A)>C_{a}$ when $F(A)=a$. To see this, consider an arbitrary point $\lambda_{0} \in \partial \Gamma$ satisfying $\sup _{t>0} f\left(\lambda_{0}+\right.$ $t \gamma)>a+1$, where $\gamma$ is the inner unit normal of $\partial \Gamma$, or a proper unit vector at $\lambda_{0}$, pointing to the interior of $\Gamma$ if $\partial \Gamma$ is not $C^{1}$ at $\lambda_{0}$. Let $t_{a}>0$ such that $f\left(\lambda_{0}+t_{a} \gamma\right)=a$. Then the derivative $\frac{d}{d t} f\left(\lambda_{0}+t_{a} \gamma\right)$ is strictly positive. Note that $\varphi u^{-k \alpha}$ is bounded. Hence $\mathcal{F} \geq C$ in (3.12). Hence (3.6) holds.

As indicated before, the above proof is essentially contained in [GW1,GW3,STW]. We include the proof for the convenience of the readers.

We will establish the gradient estimate (3.10) in the next section. Once the first and second order derivatives are bounded, the equation becomes uniformly elliptic. Hence by Evans-Krylov's regularity, we have the following regularity result. 
Theorem 3.1. Let $u \in C^{3,1}$ be a positive solution of (3.5) in $B_{r}(0) \subset \mathcal{M}$. Suppose $\varphi>0, \in C^{1,1}$. Then for any $\alpha \in(0,1)$,

$$
\|u\|_{C^{3, \alpha}\left(B_{r / 2}(0)\right)} \leq C,
$$

where $C$ depends only on $n, r, \inf _{\mathcal{M}} u, \varphi$, and $g_{0}$.

\section{Interior gradient estimate}

In this section we prove an interior gradient estimate for equation (1.11). We write equation (1.11) in the form

$$
F\left(\nabla_{c o n f}^{2} v+A v\right)=\varphi v^{\alpha \frac{n+2}{n-2}}
$$

where $A=\frac{n-2}{2} A_{g_{0}}$. Let $v$ be a solution of (4.1) in a geodesic ball $B_{r}(0) \subset \mathcal{M}$ and let

$$
z=\rho \frac{|\nabla v|}{v}
$$

be an auxiliary function, where $\rho=1-\frac{|x|^{2}}{r^{2}}$ is a cut-off function. We want to prove that $z$ is uniformly bounded, so that $(2.1)$ holds

If estimate (2.1) is not true, there is a sequence of solutions $v_{k}$ such that $\sup z_{k} \rightarrow \infty$, where $z_{k}=\rho \frac{\left|\nabla v_{k}\right|}{v_{k}}$. Assume $\sup z_{k}$ is attained at $x_{k}$. We may assume

$$
v_{k}\left(x_{k}\right)=1
$$

for otherwise we may replace $v_{k}$ by $\hat{v}_{k}=\frac{v_{k}}{v_{k}\left(x_{k}\right)}$. Then $\hat{v}_{k}$ satisfies

$$
F\left(\nabla_{c o n f}^{2} v+A v\right)=\bar{c}_{k} \varphi v^{\alpha \frac{n+2}{n-2}}
$$

where $\bar{c}_{k}=\left(v_{k}\left(x_{k}\right)\right)^{4 \alpha /(n-2)}$ and $\alpha>0$ is the homogeneity constant in (f5). Note that our estimate allows $\sup z$ depends on $\sup v$. Hence $\bar{c}_{k}$ is bounded above.

Denote $d_{k}=r-\left|x_{k}\right|=\operatorname{dist}\left(x_{k}, \partial B_{r}(0)\right)$. We have

$$
d_{k}\left|\nabla v_{k}\left(x_{k}\right)\right| \geq r \sup z_{k} \rightarrow \infty
$$

Choose a normal coordinate at $x_{k}$ and identify $B_{r}\left(x_{k}\right)$ with a Euclidean ball $B_{r}^{e}(0)$ by the exponential map. Make the dilation

$$
y=x\left|\nabla v_{k}\left(x_{k}\right)\right|
$$

Then $v_{k}$ is defined in a ball $B^{e}(0)$ of radius

$$
r_{k}:=d_{k}\left|\nabla v_{k}\left(x_{k}\right)\right| \rightarrow \infty
$$


and satisfies the equation

$$
F\left(\nabla_{c o n f}^{2} v+A_{k} v\right)=c_{k} \varphi v^{\alpha \frac{n+2}{n-2}}
$$

where $A_{k}=A\left|\nabla v_{k}\left(x_{k}\right)\right|^{-2}$ and $c_{k}=\left|\nabla v_{k}\left(x_{k}\right)\right|^{-2 \alpha}$. Moreover,

$$
v_{k}=1 \quad\left|\nabla v_{k}\right|=1 \quad \text { at } y=0 .
$$

Since $\sup z_{k}$ is attained at $y=0$ and note that $\rho>\frac{1}{2} \rho\left(x_{k}\right)$ when $\left|x-x_{k}\right|<\frac{1}{2} d_{k}$, we see that $\frac{\left|\nabla_{y} v_{k}\right|}{v_{k}} \leq 2$ for $y \in B_{r_{k} / 2}^{e}(0)$. Hence $v$ and $v^{-1}$ are locally uniformly bounded. Therefore for any $R>0$, by the interior second derivative estimate, $v_{k}$ is uniformly bounded in $C^{1,1}\left(B_{R}^{e}(0)\right)$ provided $k$ is sufficiently large such that $\frac{r_{k}}{2}>R$. Hence by the Arzela-Ascoli lemma, there is a subsequence of $v_{k}(y)$ which converges to a limit function $v \in C^{1,1}\left(\mathbf{R}^{n}\right)$, and $v$ is a solution of

$$
F\left(\nabla_{c o n f}^{2} v\right)=0
$$

Hence by $[\mathrm{L}]$, we have $v \equiv 1$. On the other hand, by (4.4) and the interior second derivative estimate, we have $|\nabla v|=1$ at $y=0$. We reach a contradiction and thus proved the interior gradient estimate.

\section{Existence of solutions}

The existence of solutions to equation (1.1) for general $f$ has been studied in [GV1,LL2] and [TW2], where the equation need not to be variational. The key assumption is the interior a priori estimates, which were established previously for the conformal $k$ Hessian equations and their quotient equations (see [GW1]). With our a priori estimates (Theorems 2.1 and 2.2) for general $f$ (see also [Ch]), we can prove the existence of solutions to (1.11) as in [LL1, TW2], as stated in Theorems 2.3 and 2.4. So we outline the proof here.

The proof of Theorem 2.3 is already in [LL1] (assuming the a priori estimates, Theorems 2.1 and 2.2). Indeed, it suffices to prove that the solution is uniformly bounded, namely

$$
\sup v \leq C
$$

for some $C$ depending only on $\left(\mathcal{M}, g_{0}\right)$ but independent of $f$. We point out the main idea here. Let $\tilde{\mathcal{M}}$ be the universal cover of $\mathcal{M}$, with the pull back of $g_{0}$ as the metric. Then the function $\tilde{v}$ on $\tilde{M}$, determined by $v$, is also a solution to (1.11). If $\tilde{\mathcal{M}}$ is the unit sphere, by the stereographic projection, $\tilde{\mathcal{M}}$ (after taking away one point) is conformally equivalent to the Euclidean space. Hence by the Liouville Theorem [LL2], $\tilde{v}$ has a unique maximum point. Hence $\sup v$ is bounded. 
If $\tilde{\mathcal{M}}$ is not the unit sphere, it is conformal to a domain $\tilde{\Omega} \subset S^{n}, \neq S^{n}[\mathrm{SY}]$. Using the stereographic projection, $\tilde{\mathcal{M}}$ is conformal to a domain $\Omega \subset \mathbf{R}^{n}, \neq R^{n}$. By the moving plane argument [Ye], the gradient of $\tilde{v}$ is uniformly bounded, $|\nabla \tilde{v}| \leq C$ for some $C$ depending only on $\Omega$ (independent of $f$ ). Hence $v$ is uniformly bounded on $\mathcal{M}$.

By (5.1) and Theorems 2.1 and 2.2, we obtain

$$
\|v\|_{C^{2, \alpha}}+\left\|v^{-1}\right\|_{C^{2, \alpha}} \leq C
$$

Hence by a degree argument, we obtain a solution of (1.11).

For the proof of Theorem 2.4, we proceed as in [TW2]. We have a stronger result, that is

Theorem 5.1. Let $\left(\mathcal{M}, g_{0}\right)$ be a compact $n$-manifold not conformally equivalent to the unit sphere $S^{n}$. Suppose $\Gamma \subset \Sigma_{\delta}$ for some $\delta<\frac{1}{n-2}$. Then the set $\left[g_{0}\right]_{\Gamma}$ is compact in $C^{0}(\mathcal{M})$ and satisfies the following Harnack inequality, namely for any $g=e^{-2 w} g_{0} \in$ $\left[g_{0}\right]_{\Gamma}$,

$$
|w(x)-w(y)| \leq C|x-y|^{\beta}
$$

for some constant $C$ depending only on $\Gamma$ and $\left(\mathcal{M}, g_{0}\right)$, independent of $f$, where $\beta=$ $\frac{1-\delta(n-2)}{1+\delta}$ and $|x-y|$ denotes geodesic distance in the metric $g_{0}$ between $x$ and $y$.

The compactness above is understood in the sense that $c g$ is regarded as the same metric $g$ for any positive constant $c$. Theorem 2.4 follows immediately from Theorem 5.1 by a degree argument [TW2], and the proof of Theorem 5.1 is also similar to that of Theorem A in [TW2]. We outline the main steps here.

(i) For any metric $g=e^{-2 w} g_{0} \in\left[g_{0}\right]_{\Gamma}$, by subtracting a linear function we may assume $\sup w=0$. By the assumption $\Gamma \subset \Sigma_{\delta}$, one can show $u=e^{w}$ is uniformly Holder continuous with Holder exponent $\beta=\frac{1-\delta(n-2)}{1+\delta}$.

(ii) If (5.3) is not true, there is a sequence of metrics $g_{k}=e^{-2 w_{k}} g_{0} \in\left[g_{0}\right]_{\Gamma}$ such that $\inf w_{k} \rightarrow-\infty$. Let $w=\lim w_{k}$. By (i), $w$ is singular at some points. Let $\tilde{w}(r)=$ $\inf \left\{h \mid B_{r}(0) \subset\{w<h\}\right\}$ be the least radial function satisfying $\tilde{w} \geq w$. We show that either $\tilde{w}$ is Hölder continuous or

$$
\tilde{w}(r)=2 \log r+c+o(r)
$$

(iii) In the case (5.4), by a blow-up argument and the comparison principle, we have

$$
w(x)=2 \log |x|+c+o(1)
$$

Furthermore $w$ has isolated singularities.

(iv) By (5.5) we prove that $w$ has at most one singular point. Indeed, if $g \in\left[g_{0}\right]_{\Gamma}$, the Ricci curvature $R i c_{g} \geq 0$, as indicated before Theorem 2.4. Hence the ratio $Q(r)=\frac{\operatorname{Vol}\left(B_{y, r}[g]\right)}{\operatorname{Vol}\left(B_{y, r}^{e}\right)}$ 
is decreasing, where $B_{y, r}[g]$ denotes geodesic ball in the metric $g$ and $B_{y, r}^{e}$ denotes ball in the Euclidean space. Hence

$$
Q(r) \leq 1
$$

By (5.5), we have

$$
Q(r) \rightarrow m
$$

as $r \rightarrow \infty$, where $m$ is the number of singular points. Hence $m=1$ and

$$
Q(r) \equiv 1 .
$$

(v) By (5.6) we show that if $w$ has a singular point $\{0\}$, then $w \in C^{\infty}(\mathcal{M} \backslash\{0\})$. Indeed, for any point $y \in \mathcal{M} \backslash\{0\}$, consider the Perron lifting of $w$ in $B_{y, r}$, that is the function $w^{*}$ given by

$$
\begin{aligned}
& \sigma_{k}\left(\lambda\left(w^{*}\right)\right)=0 \text { in } B_{y, r} \\
& w^{*}=w \text { in } \mathcal{M} \backslash\left\{B_{y, r}\right\}
\end{aligned}
$$

Then $w^{*}$ is admissible and $w^{*} \geq w$. By (5.6) we conclude $w=w^{*}$ and so $w \in C^{1,1}$. By (5.6) we can show furthermore that the scalar curvature $R_{g}=0$. Hence $w \in C^{\infty}(\mathcal{M} \backslash\{0\})$. (vi) It follows that $(\mathcal{M} \backslash\{0\}, g)$ is a complete smooth manifold with $\operatorname{Ric}_{g} \geq 0$ and $Q(r) \equiv$ 1. Hence it is isometric to $\mathbf{R}^{n}$, which in turn implies that $\mathcal{M}$ is conformal to $S^{n}$, a contradiction.

Having established the estimate (5.3), one can prove the existence of solutions of (1.11) by a degree argument. We remark that under the conditions in Theorem 5.1, there is a solution to

$$
\left.F\left(\nabla_{c o n f}^{2} v+\frac{n-2}{2} v A_{g_{0}}\right)\right)=\varphi v^{p}
$$

for any $p>\alpha \frac{n+2}{n-2}$. Hence there is no critical exponent in this case.

Remark 5.1. As in [TW2], one can prove that if $\Gamma \subset \Sigma_{\delta}$ for some $0 \leq \delta<\frac{1}{n-2}$, and if $g=v^{\frac{4}{n-2}} g_{0}$ is an admissible metric on $\mathbf{R}^{n}$, then either $v$ is Holder continuous or

$$
v(x)=C|x|^{2-n} .
$$

Remark 5.2. Our assumption on $f$ is stronger than that in [L], except that we drop the smoothness condition $f \in C^{1}$ in $[\mathrm{L}]$, so that it embraces example (ii). In the proof of the Liouville theorem $[\mathrm{L}]$, the smoothness $f \in C^{1}$ is not required, the local Lipschitz continuity of $f$, which follows from the concavity of $f$, is enough. Alternatively, one can also use smooth functions to approximate $f$, as the estimates (5.1) and (5.3) are independent of $f$. We also remark that if $v$ is an admissible solution with bounded second derivatives, equation (1.11) is uniformly elliptic and so $v \in C^{2, \alpha}[\mathrm{GT}]$. 


\section{REFERENCES}

[A] T. Aubin, Some nonlinear problems in Riemannian geometry, Springer, 1998.

[CNS] L.A. Caffarelli, L. Nirenberg, and J. Spruck, Dirichlet problem for nonlinear second order elliptic equations III. Functions of the eigenvalues of the Hessian, Acta Math. 155(1985), 261-301.

[CGY] A. Chang, M. Gursky, P. Yang, An a priori estimate for a fully nonlinear equation on four-manifolds, J. Anal. Math. 87 (2002), 151-186.

[Ch] S. Chen, Local estimates for some fully nonlinear elliptic equation, preprint of September 2005.

[CW] K.S. Chou and X-J. Wang, A variational theory of the Hessian equation, Comm. Pure Appl. Math. 54 (2001), 1029-1064.

[G] C. Gerhardt, Closed Weingarten hypersurfaces in Riemannian manifolds, J. Diff. Geom., 43(1996), 612-641.

[GT] D. Gilbarg and N.S. Trudinger, Elliptic partial differential equations of second order, Springer, 1983.

[GW1] P. Guan and G. Wang, Local estimates for a class of fully nonlinear equations arising from conformal geometry, Int. Math. Res. Not. (2003), 1413-1432.

[GW2] P. Guan and G. Wang, A fully nonlinear conformal flow on locally conformally flat manifolds, J. Reine Angew. Math. 557 (2003), 219-238.

[GW3] P. Guan and Xu-Jia Wang, On a Monge-Ampère equation arising in geometric optics, J. Diff. Geom., 48(1998), 205-223.

[GV1] M. Gursky and J.Viaclovsky, Prescribing symmetric functions of the eigenvalues of the Ricci tensor, Ann. Math., to appear.

[GV2] M. Gursky and J.Viaclovsky, Convexity and singularities of curvature equations in conformal geometry, arXiv:math.DG/0504066.

[I] N. Ivochkina, Solution of the Dirichlet problem for some equations of Monge-Ampère type. Mat. Sb., 128(1985), 403-415.

[Kr] N.V. Krylov, On the general notion of fully nonlinear second-order elliptic equations, Trans. Amer. Math. Soc., 347(1995), 857-895.

[LL1] A. Li and Y.Y. Li, On some conformally invariant fully nonlinear equations. Comm. Pure Appl. Math. 56 (2003), 1416-1464.

[LL2] A. Li and Y.Y. Li, On some conformally invariant fully nonlinear equations II, Liouville, Harnack, and Yamabe, Acta Math., to appear.

[L] Y.Y. Li, Degenerate conformal invariant fully nonlinear elliptic equations, arXiv:math.AP/0504598.

[STW] W.M. Sheng, N.S. Trudinger, X.-J. Wang, The Yamabe problem for higher order curvatures, arXiv:math.DG/0505463.

[SY] R. Schoen and S.T. Yau, Lectures on Differential geometry. International Press, 1994.

[TW1] N.S. Trudinger and X-J. Wang, Hessian measures II, Ann. of Math. (2) 150 (1999), 579-604.

[TW2] N.S. Trudinger and X-J. Wang, On Harnack inequalities and singularities of admissible metrics in the Yamabe problem, arXiv:math.DG/0509341.

[V] J. Viaclovsky, Estimates and existence results for some fully nonlinear elliptic equations on Riemannian manifolds, Comm. Anal. Geom. 10 (2002), 815-846.

[Ye] R. Ye, Global existence and convergence of Yamabe flow. J. Differential Geom. 39 (1994), 35-50.

Centre for Mathematics and its Applications, Australian National University, Canberra ACT 0200, Australia

E-mail address: wang@maths.anu.edu.au 